\title{
Reconstruction of Crime Scene, a Forensic Technique used in Substantiating Alleged Homicide into Accidental Death: A Case Study
}

\section{Kumar $\mathbf{A}^{1^{*}}$ and Goyal $\mathbf{M K}^{2}$}

${ }^{1}$ Assistant Director (Scene of Crime), Forensic Science Laboratory (Haryana) Madhuban, Karnal, India

${ }^{2}$ Former Deputy Director, Forensic Science Laboratory (Haryana) Madhuban, Karnal, India

"Corresponding author: Ajay Kumar, Assistant Director (Scene of Crime), Forensic Science Laboratory (Haryana) Madhuban, Karnal, India, Tel: +918814874062; Email: ajayadsoc@gmail.com

Received date: August 9, 2017; Accepted date: August 17, 2017; Published date: August 25, 2017

Copyright: (c) 2017 Kumar A. This is an open-access article distributed under the terms of the Creative Commons Attribution License, which permits unrestricted use, distribution, and reproduction in any medium, provided the original author and source are credited.

\begin{abstract}
Crime scene reconstruction is the process of determining or eliminating the events that occurred at the crime scene by analysis of physical evidences. Through this paper we will discuss a case of accident that was turned into murder (i.e. Under Section 302 of Indian Penal Court) because the family members of the deceased alleged that the deceased was murdered and then dragged by tying to a vehicle to dispose off the body and to destroy the evidences. In this paper we discuss how this sensational case was solved by the scientists of Forensic Science Laboratory, Haryana through the reconstruction of crime scene by analyzing crime scene appearance, the locations and positions of the physical evidence, and the injuries to the deceased. This involved scientific crime scene investigation, interpretation of patterned evidence at the scene, laboratory testing of the physical evidence, systematic study of related case information, and the logical formulation of a theory.
\end{abstract}

Keywords Crime scene; Reconstruction; Homicide; Physical evidence; Injury

\section{Material and Methods}

The Crime scene was reconstructed on the bases of scientific experimentation and experience of the forensic scientists. Its steps and stages followed basic scientific principles, theory formulation and logical methodology. During the reconstruction all investigative information with physical evidence analysis and interpretation incorporated which was molded into a reasonable explanation of the activity and related events.

During this logic, careful observation, and considerable experience, both in crime scene investigation and forensic testing played a critical role. All data concerning the condition of physical evidence, patterns and impressions, condition of the victim, etc., was reviewed, organized, and studied.

\section{Observations recorded from the photographs: Figure 1}

- Abraded area on the right side part of the skull exposing the underling bone. No damage on the earlobe, cheek, mandible, lips and neck was observed (Photograph 1).

- No noticeable damage on the face, front of neck, forehead, nose, and upper part of the chest could be observed (Photograph 2).

- No noticeable damage on the right hand including phalanges, palmer area. Little damage present on the palm towards wrist. No noticeable damage on the left side of head, earlobe, eye, cheek, nose and shoulder etc. Superficial injuries are seen on thex upper area of left arm (Photograph 3).

- Left hand phalanges, palmer area and wrist escaped injuries (Photograph 4).
- Injuries/road side injuries on the back and on the right hip region. There is darkish demarcated stained area on the lower area of right leg. No damage seen on the left leg region (Photograph 5).

- Abraded damage with tags of flesh on the inner area of heal and phalanges of the left foot. Damage was observed on the fifth toe of the right foot.

- Superficial damage/abrasion on the outer area of right forearm towards elbow (Photograph 6).

\section{From the damages caused on the body as seen in the photographs following inferences are drawn:}

- The deceased received injuries on its body mainly on the right side part i.e. back of skull, back of body, hip, and left foot.

- Right foot and both the legs had insignificant damages/injuries indicated thereby that the body was elevated from the leg side and was touching the road from head side with its back.

- The damages on the left foot and right foot are not similar i.e. the left foot was in contact with the road while the right foot was not.

- Absence of injuries on the face, chest, hands and insignificant injuries on the arms indicate that the deceased was having some control over the body while being dragged on the road.

\section{Observations recorded from the post mortem report}

- Eighteen injuries have been recorded in the PMR out of which sixteen are ante mortem.

- The post mortem injuries are only left side of right knee and on the front of the right upper arm.

- The ante mortem injuries are mostly on the right side and back of the body and are grazed abrasions. No significant damage recorded on the legs.

- Injury no 14 comprises reddish contusion of size $7 \times 2 \mathrm{~cm}$ on the right lower leg on the posterior medial region. 
Citation: Kumar A, Goyal MK (2017) Reconstruction of Crime Scene, a Forensic Technique used in Substantiating Alleged Homicide into Accidental Death: A Case Study. J Forensic Res 8: 387. doi:10.4172/2157-7145.1000387

Page 2 of 3

- Injury no 15 comprises an abrasion on the right foot of 5th finger toe on the lateral part size $3 \times 1 \mathrm{~cm}$ reddish and blackish colour.

- Injury no 5 comprises of a lacerated wound of size $4 \times 3 \mathrm{~cm}$ on the planter side (sole) of left foot on the heel region reddish black graze marks in the diameter of $6 \mathrm{~cm}$. Lacerated wound of size $3 \times 2 \mathrm{~cm}$ on the planter side of left first (great) toe of foot with reddish black graze abrasions in the diameter of $3 \mathrm{~cm}$.
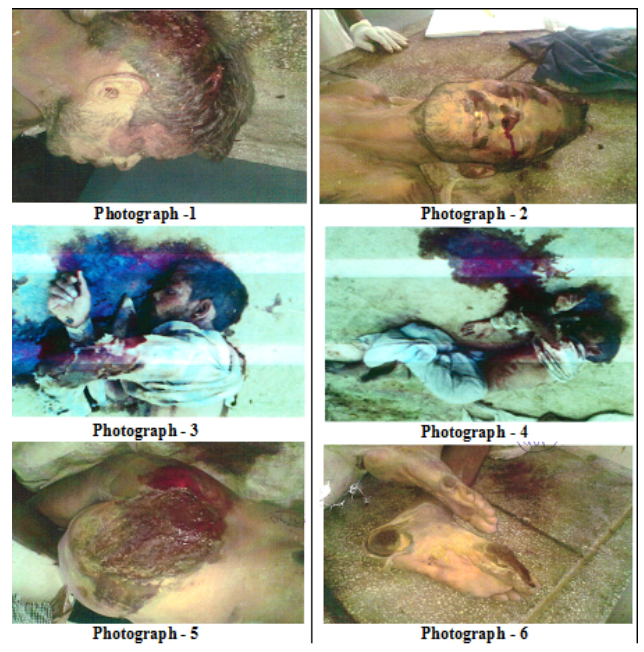

Figure 1: Observations recorded from the photographs.

\section{From the injuries recorded in the PMR it is inferred that}

- The deceased received injuries while being dragged from the leg side with his back side of head, back, right hip and left foot touching the road.

- The lower part of the right leg on the posterior medial region was touching some smooth and hard surface.

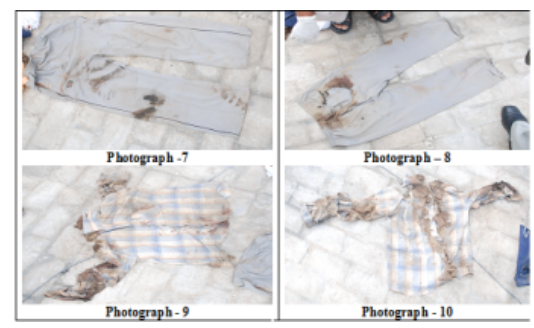

Figure 2: Injuries recorded in PMR.

\section{Observations recorded from the clothes of the deceased: Figure 2}

Clothes of deceased were examined in the custody of the police and following observations were made:

- Trousers/lower of the deceased had graze marks with blood and a hole and tags of torn out cloth on the right back hip region (Photograph 7and8).
- Oily blackish stains/smears were present on the lower margin of the right leg of the trousers. No tearing or graze marks were observed on any other part of the trousers except the hip region.

- The shirt was torn with tags of cloth stained with blood on its back. The front of the shirt was totally devoid of any damage/graze mark. Buttons of the shirt were intact and normal. Collar on the back right side area was torn and stained with blood (Photograph 9and10).

From the observations recorded from the cloths of deceased, it is referred that

- That the deceased received injuries/damages while being dragged on its back.

- The right leg appeared to have been in contact with some object having oily blackish surface.

\section{Observations made at the scene of crime: Figure 3}

- The alleged place/kotha were located along the road leading from village Nand Garh to Gohana via village Bhavad (Photograph 11).

- No marks of any vehicle or any drag marks except an alleged darkish stain of blood could be observed on the road in front of the 'kotha'. The alleged stain of blood was tested and was found to be negative for blood.

- After a distance of about $500 \mathrm{~m}$ a hand pump was observed on the bank of 'rajwaha' on the right side of the road towards Bhavad (Photograph 12).

- After a distance $1100 \mathrm{~m}$ drag marks leading towards village Bhavad were observed on the road and were found positive for blood (Photograph 13).

- A liquor shop was found approximately $3000 \mathrm{~m}$ from the 'kotha' towards Bhavad. A speed breaker and a dug out channel by removing the concrete tiles across the road were observed just before the place from where dead body was recovered (Photograph 14).

- A large size darkish stain of blood was observed on the spot in front of the dairy.

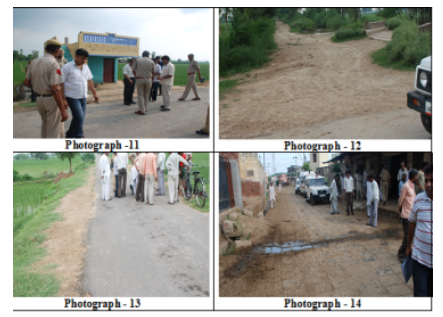

Figure 3: Observations made at the scene of crime.

\section{Inferences}

On the basis of observations made at the scene of crime and recorded from the PMR and the photographs, it is inferred as under:

- The deceased Bijender Singh was alive at the time of receiving injuries while being dragged.

- The body of the deceased was dragged from leg side with his head side dragged against the road. 
Citation: Kumar A, Goyal MK (2017) Reconstruction of Crime Scene, a Forensic Technique used in Substantiating Alleged Homicide into Accidental Death: A Case Study. J Forensic Res 8: 387. doi:10.4172/2157-7145.1000387

Page 3 of 3

- The left leg of the deceased was in contact of the road and the right leg was away from the road while the body was being dragged.

- The lower part of right leg and right foot were in contact with some hard object having smooth and oily blackish surface.

\section{Opinion}

On the basis of the inferences drawn above it is opined that the deceased Sh. Bijender Singh was dragged by a moving vehicle from leg side to head side while alive and was having some control on his body.

\section{Conclusion}

On the basis of observation recorded, inferences drawn opinion made and narrations made by the police and the inmates of the deceased it appears as if the deceased under intoxication could have requested a vehicle to stop to fetch something and could have tried to board the same from back side taking his first step using his right foot which accidentally could have slipped inside some gap and got entangled and lost control over the body and was dragged accidentally by the driver of the vehicle. After covering a distance of $3 \mathrm{~km}$ on the clear metalled road vehicle could have received a jerk while crossing the speed breaker and the dug out part of the road and the body could have been released on the road/spot from where it was recovered.

\section{References}

1. Fisher BAJ (1993) Techniques of crime scene investigation (5th edtn). CRC Press Inc, Florida, USA.

2. Dix J (1999) Handbook for death scene investigators (2nd edtn) CRC press, Florida, USA.

3. Vernon JG (1996) Practical homicide investigation checklist and field guide (2nd edtn), CRC press, Newyork, USA.

4. Vallejo CA, Edsicker D (2001) Crime scene investigation and physical evidence manual.

5. Sharma BR (2004) Forensic science and criminal investigation techniques.

6. Dogra TD, Rudra A (2005) Lyons: Medical jurisprudence and toxicology. (11th edtn), New Delhi, India. 